\title{
Correlation, Entanglement and Locality of Quantum Theory
}

\author{
S.V.Gantsevich* and V.L.Gurevich \\ Ioffe Institute, Russian Academy of Sciences, 194021 Saint Petersburg, Russia \\ Email: sergei.elur@mail.ioffe.ru
}

\begin{abstract}
In recent decades it was established that the measurements of physical quantities in space-time points divided by large space-like intervals can be correlated. The correlation is created by the measurements while their mutual influence requires the corresponding supraliminal velocities. This situation is commonly known as the violation of the Bell inequality in the Bohm version of Einstein-Podolsky-Rosen paradox. Nearly 40 years the question remains disputable with a variety of contradicting views. The most frequently the violation is explained by the existence of some momentary action at a distance which obliges the quantum mechanics (as well as all nature) to be nonlocal. We find the "common cause in the past" as the origin of the observed quantum correlation which so far was unnoticed either by the partisans of quantum non-locality or their opponents. The novelty of our approach is the accent on the expressions for the observed physical quantities rather than on the "entangled" two-particle wave functions. We show the crucial role of phases in the correlation process that is hard to notice using the traditional approach.
\end{abstract}

Keywords: Quantum correlation, entangled states, bell inequality, EPR-paradox

\section{Introduction}

At first let us note that quantum particles can be correlated without any interaction between them. The correlation is ensured by the quantum exchange mechanism and reveals itself in a large number of physical phenomena. The microscopic consequences of the quantum exchange effects were well known but attracted little attention and the explanation reduced to the words "it was a quantum effect".

Nowadays the word "exchange" was replaced everywhere by the word "entanglement" which became extremely popular. It occurred due to the macroscopic revelation of the quantum exchange correlation the most striking result of which was the correlation of observed physical quantities divided by large macroscopic space-type intervals.

During last forty years this phenomenon was observed at very large distances (up to tens of kilometers) and therefore looked puzzling and mysterious. Generally it is observed in the form of the violation of the Bell inequality in the Bohm version of the Einstein-Podolsky-Rosen paradox.

The EPR-paradox was formulated at first by Einstein and coauthors in 1935 [1]. After the immediate Bohr reply [2] defending the QM consistency the paradox was nearly forgotten for years. In 1951 Bohm proposed its very transparent spin-version [3] and later in 1964 Bell constructed his famous inequality [4] that made possible the experimental verification of $\operatorname{EPR}(\mathrm{B})$ quantum correlation.

The experiments followed $[5,6,7]$ and gave rise to the enormous incessant flow of literature, (see, e.g. $[8,9,10,11,12,13,14,15,16])$. Recently the most careful experiment of this type and its interpretation as the final proof of the non-locality of nature was published $[17,18]$ and was intensively discussed. Nevertheless, there is no agreement now among the physical community on the meaning and physical origin of the observed correlation.

What is the reason for the mystery of the quantum correlation in the EPR-paradox? It is in the apparent contradiction between the principles of quantum mechanics and the theory of relativity what was first noted by Einstein and his coauthors. Briefly this contradiction can be formulated in the following way.

The correlation between two events (in this case they are measurements) can be either because of their mutual influence or because of some "common cause in the past". For one-time measurements the mutual influence is excluded by the absence of infinite interaction velocities stated by the relativity theory.

Then the reason for the correlation should be the common cause in the past. For the classical physical quantities such common cause can be easily found. However, in QM the situation is different. According 
to its principles the observed values of physical quantities are created during the measurements and do not exist before the measurements. It seems that any common cause in the past is out of question. The possible common cause in the past in the $\operatorname{EPR}(\mathrm{B})$ experiments in the form of the correlation of spin values existing before the measurements (in contradiction with QM!) is excluded by the observed violation of the Bell inequality.

Thus there is no mutual influence of measurements and no common cause for the correlation, but the experiments show that it exists. Just this situation is considered as puzzling and hard to explain.

The violation of the Bell inequality is frequently proclaimed by the major event of the physics of our time.

In order to overcome the explanation difficulty one frequently resorts to the mutual influence of measurements in a form of the so-called momentary "spooky action at a distance" which was earlier derided by Einstein. The existence of "entangled quantum states" with the momentary link at a distance usually is called the "non-locality of Quantum Mechanics". To avoid the contradiction with Relativity Theory the partisans of non-locality assert that RT forbids only supraluminal signals and permits the measurement momentary influence (that allegedly is inherent in QM) because of its random unpredicted character.

Thus the non-locality of QM follows from the violation of the Bell inequality while its violation is explained by the non-locality of QM and the existence of non-local entangled states of unclear physical nature.

One should emphasize that the mathematics of QM and generally of Quantum Theory is strictly local without any supraluminal interaction of any kind. The non-locality with the momentary action at a distance contradicts the traditional physical picture of point-to-point interaction and the absence of any type of action at a distance. This picture goes from the time of Faraday and Maxwell and is the basis of all our physical considerations.

Below by the usual QM means we shall find the physical common cause in the past for quantum correlation at a distance as it should be in the traditional physical picture. We shall show that the correlation cause is the phase relations among wave functions that describe the correlation effect. So far nobody could find this common cause in the past including also the opponents of the non-locality and supraluminal influence of measurements. In our approach we shall put accent on the observed physical quantities rather then on the combine two-particle wave functions. This small but very important modification is a novelty of our approach.

We shall explain the violation of the Bell inequality by the contribution of the phase correlated quantum states. We also shall show what the mysterious entangled particles really are and why they can be correlated at long distances and large time intervals without mysterious instantaneous link through the entire Universe.

\section{Quantum Exchange Correlation}

The correlation of physical quantities in space-time points divided by space-like intervals follows from the formulae of quantum mechanics. Let us note that despite the very large number of works where the expressions describing the quantum correlation were derived the common cause of the past for this phenomena has not been found. Also no mention of it was present in the works related to correlation phenomena in optics (see, e.g. [19,20]. Without the common cause in the past one is forced either to abstain of any explanation or to resort to supraluminal interaction and non-locality. This impasse situation arises because of the initial "faux pas" in the traditional approach to the problem where one always begins with the two-particle "maximally entangled" singlet wave function ignoring the phase relations between wave functions and the existence of state occupancy numbers.

However, as we shall see below the arbitrary initial phases of wave functions describing the observable quantities have crucial importance for the physics of correlation. These phases are implicitly present in the occupation numbers of the participating quantum states. But they do not enter into the final correlation expressions and their role remains unnoticed by the traditional approach.

In the correlation experiments one source of "correlated entangled particles" was used. However, the same expressions for the correlation take place for two totally independent sources. It is preferable to consider just this case where any correlation prior to measurements is excluded by definition. 
Thus let us consider two independent sources of quantum particles (bosons or fermions) and two independent detectors to register them. We assume that there is a source $U$ generating particles in the state $u$ and another source $V$ generating particles in the state $v$. Let the wave functions of these states be the eigenfunctions of the time-independent Hamiltonian $H$ so their space shape remains unchanged during the time evolution and only phases varies with time:

$$
\psi(r, t)=\exp (-i H t) \psi(r, 0)=\exp (-i \omega t) \psi(r, 0)
$$

We assume also that there are two spatially separated detectors measuring two physical quantities $A$ and $B$ and the results of measurements can be recorded and compared. For simplicity we assume that the measurements in two detectors are performed at the same time $t$.

To begin with, let us suppose that the particles from the source $U$ hit the detector $A$ while the particles from the source $V$ hit the detector $B$. Then the observable quantities $\bar{A}$ and $\bar{B}$ are given by

$$
\bar{A}=\langle u|A| u\rangle F_{u}, \quad \bar{B}=\langle v|B| v\rangle F_{v} .
$$

Here $F_{u}$ and $F_{v}$ are the state occupancies or distribution functions. They describe the intensities of the sources. All interactions before the measurements are absent and these intensities are constant in time $F(t)=F(0)$.

It is convenient to use the Dirac notation for wave functions calling ket the wave function $|\ldots\rangle \equiv \psi$ and $b r a$ the complex conjugated wave function $\langle\ldots| \equiv \psi^{\dagger}$.

Let us emphasize that to present a result of measurement of a physical quantity one needs just two items, i.e. a bra and a ket. Thus a potentially observed quantum particle should be represented by a bra + ket pair. For brevity we will call "wavicle" such (bra+ket) pair. (The term is used in the popular literature in order to accentuate the distinction between quantum particles and classical waves or point-like particles).

Thus we may say that the sources emit wavicles and the detectors register them. Note that the bra or ket functions always have arbitrary phases but to form the occupation number the bra and ket phases should be equal and opposite in sign. The phases acquired by them during their time evolution also remain equal and sign-opposite. Such (bra+ket) pairs relate to pure quantum states and may be called pure pairs. The intrinsic phases of pure (bra+ket) pairs do not appear explicitly in the expressions (1) for $\bar{A}$ and $\bar{B}$.

Now we assume that the wavicles (i.e bra+ket pairs) emitted by the sources $U$ and $V$ can reach both detectors. Then we have in each detector the sum of independent contributions from both sources:

$$
\bar{A}=\langle u|A| u\rangle F_{u}+\langle v|A| v\rangle F_{v}, \quad \bar{B}=\langle u|B| u\rangle F_{u}+\langle v|B| v\rangle F_{v}
$$

The mean product $\overline{A B}$ obtained from the expressions (2) contains no trace of correlation:

$$
(\overline{A B})_{\text {uncor }}=[\langle u|A| u\rangle\langle v|B| v\rangle+\langle v|A| v\rangle\langle u|B| u\rangle] F_{u} F_{v} .
$$

In this expression the flow of wavicles emitted by the sources looks as the flow of classical particles that hit both detectors with the probability $F_{u} F_{v}$.

Now let us take into account that two wavicles emitted by the sources are represented by two (bra+ket) pairs. Thus we actually have not two but four objects, i.e. two bra and two ket with two values of initial phases $\pm \varphi_{u}$ and $\pm \varphi_{v}$ as well as two values of acquired phases $\pm \omega_{u} t$ and $\pm \omega_{v} t$.

A new and interesting situation emerges where the bra and ket come into detectors pairwise from two different sources. In this case the wavicles that hit the detectors are not the wavicles produced by the sources. To get these new wavicles we should take either two bra or two ket of old wavicles and exchange them. (Such exchange is in full compliance with the QM rules.)

The new wavicles become phase dependent since their bra and ket have different phase values at the measurement time $t$ :

$$
\pm \phi= \pm\left(\omega_{u} t+\varphi_{u}-\omega_{v} t-\varphi_{v}\right) .
$$

One new wavicle has the phase multipliers $e^{+i \phi}$ while another wavicle has the phase multipliers $e^{-i \phi}$. Observable quantities corresponding to these new wavicles are proportional to such multipliers. The phases $\phi$ in (4) may be considered as random quantities in series of measurements so that all contributions 
from such wavicles vanish after the averaging over these series since $\overline{\exp ( \pm i \phi)}=0$. Because of this zero the exchange contributions do not change the mean detector data (2) looking as fluctuations or noise.

It is highly important that two new wavicles obtained by the exchange from two old ones should be mutually correlated since the values of their phase difference (4) are the same. In this respect they are analogous to the bra and ket which form the occupation number. And in the same way the phase multipliers vanish in the product of these correlated wavicles $e^{+i \phi} e^{-i \phi}=1$. Therefore the product of detector data related to the contribution of correlated wavicles becomes phase independent and does not vanish after the averaging. This way the joint averaging of data in both detectors makes detectable the correlation of observed physical quantities.

Making the exchange procedure in products of the matrix elements of the expression (3) as

$$
|u\rangle \leftrightarrow|v\rangle \quad\langle u| \leftrightarrow\langle v|
$$

we get the desired exchange correlation contribution

$$
(\overline{A B})_{c o r}= \pm[\langle u|A| v\rangle\langle v|B| u\rangle+\langle u|B| v\rangle\langle v|A| u\rangle] F_{u} F_{v}
$$

Note that phase $\phi$ does not enter into the final expression for the average $(\overline{A B})_{\text {cor }}$. The exchange correlation contribution is negative for fermions and positive for bosons. The total average quantity $\overline{A B}$ is the sum of the uncorrelated and correlated parts:

$$
\overline{A B}=[\langle u|A| u\rangle\langle v|B| v\rangle \pm\langle u|A| v\rangle\langle v|B| u\rangle] F_{u} F_{v}+(u \leftrightarrow v) .
$$

Thus the physical picture of correlation created by quantum particles emitted by two independent sources and registered by two detectors looks as follows. The sources produce uncorrelated quantum particles (wavicles). These wavicles produce no correlation in the measuring devices. Using the popular word "entanglement" one can say that they are not entangled. The data obtained from such particles remain the same either after separate or after joint averaging. (The separate averaging is the independent averaging of the data of each particular detector).

When two uncorrelated wavicles exchange their bra or ket they become mutually correlated new wavicles. They have equal and sign-opposite phase difference. Without external actions the phase coherence can survive for a long time and at large distances. To avoid any misunderstanding let us emphasize that any action on one of such wavicles has no effect on its correlated partner.

Just these phase correlated wavicles may be called the entangled quantum particles. The phase correlation between them arises due to equal phase values of the bra and ket which constitute the initial occupation numbers. The occupation numbers are formed before the measurements and therefore their existence may be regarded as the physical "common cause in the past" of the exchange correlation phenomena.

Note also that despite their deciding role the phases do not enter into the final expressions for the observable quantities $\bar{A}$ and $\bar{B}$ in (2) as well as $\overline{A B}$ in (7). Hence we may regard them as some sort of hidden variables.

\section{Examples}

Now let us consider the examples of such phenomena. For the intensity correlation of two wave sources (known as the Hanbury Brown-Twiss-effect [21]) the operators $A$ and $B$ are the space position operators at points $\mathbf{r}_{1}-\mathbf{r}_{2}=\mathbf{R}$ and the wave functions are the plane waves $u \Rightarrow \mathbf{p}, v \Rightarrow \mathbf{p}^{\prime}$ ( $\mathbf{p}$ and $\mathbf{p}^{\prime}$ being the wave vectors). Then the correlation is given by

$$
\overline{A B}=\left[1 \pm \cos \left(\mathbf{p}-\mathbf{p}^{\prime}\right) \mathbf{R}\right] 2 F_{\mathbf{p}} F_{\mathbf{p}^{\prime}} .
$$

Here the first term corresponds to uncorrelated wavicles creating the homogeneous background while the second term is the interference contribution of the wavicles created by the bra and ket of different sources. The expression (8) describes also the bunching of bosons and anti-bunching of fermions when the flow of them falls on a detecting screen. We see that for $\mathbf{R} \equiv\left(\mathbf{r}_{\mathbf{1}}-\mathbf{r}_{\mathbf{2}}\right) \rightarrow 0$ the fermions "avoid" each other while the bosons tend to unite. 
For the Bohm version of EPR-paradox two spin measurements are relating to two space points. Because of importance of this case for the correlation interpretation we consider it in more details.

The operators $A$ and $B$ now represent the spin measured at given directions. Such an operator is the scalar product $S$ of the spin vector $\sigma$ formed by the Pauli matrices and the unit vector $\mathbf{n}$ of a certain direction. In the polar coordinates $S$ is given by

$$
S \equiv(\mathbf{n}, \sigma)=\sin \theta \cos \varphi \sigma_{x}+\sin \theta \sin \varphi \sigma_{y}+\cos \theta \sigma_{z}
$$

The source wave functions $|u\rangle \equiv|\uparrow\rangle$ and $|v\rangle \equiv|\downarrow\rangle$ now are the eigenfunctions of $\sigma_{z}$ with the eigenvalues $s_{u}=1$ and $s_{v}=-1$.

For pure spin states only $\sigma_{z}$ contributes to the matrix elements of the operator $S$ and we have

$$
\begin{gathered}
\langle\uparrow|S| \uparrow\rangle=\cos \theta\left\langle\uparrow\left|\sigma_{z}\right| \uparrow\right\rangle=\cos \theta, \\
\langle\downarrow|S| \downarrow\rangle=\cos \theta\left\langle\downarrow\left|\sigma_{z}\right| \downarrow\right\rangle=-\cos \theta .
\end{gathered}
$$

For the mixed states only $\sigma_{x}$ and $\sigma_{y}$ give results so we have:

$$
\begin{gathered}
\langle\uparrow|S| \downarrow\rangle=\cos \varphi \sin \theta\left\langle\uparrow\left|\sigma_{x}\right| \downarrow\right\rangle+\sin \varphi \sin \theta\left\langle\uparrow\left|\sigma_{y}\right| \downarrow\right\rangle=e^{i \varphi} \sin \theta, \\
\langle\downarrow|S| \uparrow\rangle=\cos \varphi \sin \theta\left\langle\downarrow\left|\sigma_{x}\right| \uparrow\right\rangle+\sin \varphi \sin \theta\left\langle\downarrow\left|\sigma_{y}\right| \uparrow\right\rangle=e^{-i \varphi} \sin \theta .
\end{gathered}
$$

Let the directions of spin measurements of the detectors $A$ and $B$ be given by the unit vectors a and b. Then using (9) and (10) for the matrix elements and following (2) we get:

$$
\begin{aligned}
& \bar{A}=\langle\uparrow|A| \uparrow\rangle F_{\uparrow}+\langle\downarrow|A| \downarrow\rangle F_{\downarrow}=\cos \theta_{a}\left(F_{\uparrow}-F_{\downarrow}\right), \\
& \bar{B}=\langle\uparrow|B| \uparrow\rangle F_{\uparrow}+\langle\downarrow|B| \downarrow\rangle F_{\downarrow}=\cos \theta_{b}\left(F_{\uparrow}-F_{\downarrow}\right) .
\end{aligned}
$$

Note that for $F_{\uparrow}=F_{\downarrow}$ the mean values of $\bar{A}$ and $\bar{B}$ are zero irrespective of the measurement directions. For the quantity $\overline{A B}$ as before we have two contributions. The first one is given by (6)

$$
(\overline{A B})_{\text {uncor }}=-\cos \theta_{a} \cos \theta_{b} 2 F_{\uparrow} F_{\downarrow}
$$

This expression describes the contribution of uncorrelated wavicles of two sources. The contribution of correlated (entangled) wavicles is given by the expression (7):

$$
(\overline{A B})_{\mathrm{cor}}=\mp \cos \left(\varphi_{a}-\varphi_{b}\right) \sin \theta_{a} \sin \theta_{b} 2 F_{\uparrow} F_{\downarrow}
$$

Finally for the spin correlation we have

$$
\overline{A B}=-\cos \gamma 2 F_{\uparrow} F_{\downarrow}
$$

where $\gamma$ is the angle between the measured spin directions $\mathbf{a}$ and $\mathbf{b}$ :

$$
\cos \gamma=\cos \theta_{a} \cos \theta_{b}+\cos \left(\varphi_{a}-\varphi_{b}\right) \sin \theta_{a} \sin \theta_{b}
$$

We see that the sum of two contributions becomes independent of the direction of the spin vector $\sigma$ being proportional to the scalar product of the unit vectors $\mathbf{a}$ and $\mathbf{b}$ which are the directions of independent spin observations in two space points in one time moment.

Now when we understand the physical cause of quantum correlation, the dependence (14) does not look puzzling and mysterious even though the measurement directions $\mathbf{a}$ and $\mathbf{b}$ are arbitrary and according to the quantum mechanics the detected spin values do not exist before the measurements.

For the case where $\overline{A B}$ is the matrix element $\langle\Psi|A B| \Psi\rangle$ with the singlet wave function $\Psi$, namely

$$
\Psi=\left|\uparrow_{a}\right\rangle\left|\downarrow_{b}\right\rangle-\left|\uparrow_{b}\right\rangle\left|\downarrow_{a}\right\rangle
$$

we have

$$
\overline{A B}=\langle\Psi|A B| \Psi\rangle, \quad \bar{A}=\langle\Psi|A| \Psi\rangle=0, \quad \bar{B}=\langle\Psi|B| \Psi\rangle=0
$$


that corresponds to our result of two fully independent sources under condition

$$
F_{u} \equiv F_{\uparrow}=F_{\downarrow} \equiv F_{v}=1 .
$$

We see that the singlet wave function (16) actually describes the flow of oppositely polarized spins of equal intensities. This equality is a unique property of the measurements described by matrix elements with the singlet wave function $\Psi$.

One should also remember that the correlation is of statistical nature and to observe it a series of measurements are required. The law

$$
\bar{S}=\langle\uparrow|S| \uparrow\rangle F_{\uparrow}=\cos \theta F_{\uparrow}
$$

for a flow of polarized spins in the $z$ direction measured at the angle $\theta$ can be realized as random series of positive and negative units that appear with the probabilities depending of $\theta$ :

$$
\cos \theta=(+1) \cos ^{2} \theta / 2+(-1) \sin ^{2} \theta / 2=\cos ^{2} \theta / 2-\sin ^{2} \theta / 2 .
$$

As we see from (11) for two spin flows of the opposite signs the mean observed value is proportional to the difference of intensities of the flows:

$$
\bar{S}=\langle\uparrow|S| \uparrow\rangle F_{\uparrow}+\langle\downarrow|S| \downarrow\rangle F_{\downarrow}=\cos \theta\left(F_{\uparrow}-F_{\downarrow}\right) .
$$

The observation of spins in the singlet states gives zero value for any measurement direction just because of the parity of intensities $F_{\uparrow}=F_{\downarrow}$ and is not due to the special properties of the singlet state. For $\theta=\pi / 2$ the detector always gives mean zero irrespective of the flow intensity. In this case there will be equal average number of positive and negative units fixed by the detector.

Now let us see how the Bell inequality is violated by the quantum exchange correlation described above for the case (17). We choose two directions $\mathbf{a}$ and $\mathbf{a}^{\prime}$ of the detector $A$ and two directions $\mathbf{b}$ and $\mathbf{b}^{\prime}$ of the detector $B$. Now suppose that all vectors lay in the plane $(z, x)$. The vectors $\mathbf{a}$ and $\mathbf{b}$ are parallel and $\theta_{a}=\theta_{b}=0$. The vectors $\mathbf{a}^{\prime}$ and $\mathbf{b}^{\prime}$ are mutually orthogonal and $\theta_{a^{\prime}}=-\theta_{b^{\prime}}=\pi / 4$. The Bell sum of $A B$ spin data correlation where the spin values $A$ and $B$ are always equal to \pm 1 is given by

$$
\left|A\left(B+B^{\prime}\right)+A^{\prime}\left(B-B^{\prime}\right)\right|=\left|A B+A B^{\prime}+A^{\prime} B-A^{\prime} B^{\prime}\right|=|1+1+1-1|=2
$$

According to the expressions (14)-(15) we have for chosen detector directions the corresponding quantummechanical expression:

$$
\left|\cos \gamma_{a b}+\cos \gamma_{a b^{\prime}}+\cos \gamma_{a^{\prime} b}-\cos \gamma_{a^{\prime} b^{\prime}}\right|=|1+1 / \sqrt{2}+1 / \sqrt{2}-0|=1+\sqrt{2}>2
$$

Note that in this expression the values of first three terms come only from the uncorrelated part in (14) because of $\sin \theta_{a}=\sin \theta_{b}=0$ and the correlated part is absent. The value of fourth term $\cos \left(\gamma_{a^{\prime} b^{\prime}}\right)=\cos (\pi / 2)=0$ is the sum of the both parts of (14) which cancel each other. If we exclude the correlated contribution then the sum will satisfy the Bell inequality: $|1+1 / \sqrt{2}+1 / \sqrt{2}-1 / 2|=1 / 2+\sqrt{2}<2$. We see that the Bell inequality is violated just because of the presence of the mutually correlated fluctuation contributions in both detectors.

In conclusion let us note that the exchange contribution is the natural consequence of the linearity of the equation of motion for wave functions and the bi-linearity of expressions for the observable physical quantities. This feature of QM leads to the apparition of two types of quantum states (or different bra+ket pairs). The pure states (bra and ket pairs with the same quantum indices) always give permanent contributions to physical observable quantities while mixed states (bra and ket pairs where quantum indices are different) give time and phase dependent contributions to the observable quantities. Two mixed pairs created by exchange from two pure pairs become phase correlated automatically. They are mysterious entangled particles that are so popular now.

The contributions of correlated mixed bra+ket pairs inevitably are presented in all measurements of physical quantities in any many-body system looking as correlated noise. Actually in the recent experiment of Hensen et al. [17] as well as in earlier series of similar experiments just this correlated noise was measured at the macroscopic distances. One should emphasize that the so-called nonlocality of this noise 
is caused by the local common cause in the past discussed above and not by an imaginary momentary action at a distance frequently mentioned in the literature (see, e.g. [18]). Quantum Mechanics and generally Quantum Theory are local theories and the results of these experiments give no arguments to renounce the traditional physical picture of point-like interaction and do not support the ideas of any type of supraluminal interaction.

Finally, let us note that it is interesting and instructive to compare our approach to the entanglement and locality with the ideas put forward by G.'t Hooft in his works about Quantum Mechanics (see, e.g. $[22,23])$.

\section{References}

1. A. Einstein, B.Podolsky, N.Rosen, Phys. Rev., 47, 777 (1935).

2. N.Bohr, Phys. Rev., 48, 696 (1935).

3. D.Bohm and Y.Aharonov, Phys. Rev. 108, 1070 (1957).

4. J.S.Bell, Physics 1, 195, (1964)

5. A.Aspect, P.Grangier, G.Rodger, Phys. Rev. Lett., 49, 91 (1982).

6. A. Aspect, J.Dalibard, G.Rodger, Phys. Rev. Lett., 49, 1804(1982).

7. G.Weihs et al., Phys. Rev. Lett. 81, 5039 (1998)

8. N.Brunner, D.Cavalcanti, S.Pironio, V.Scarani, S.Wehner, Rev. Mod. Phys. 86, 419 (2014).

9. M.Navascues and D.Peres-Garcia, Phys. Rev. Lett., 109, 160405 (2012).

10. S.P.Walborn, A.Salles, R.M.Gomes et al., Phys. Rev. Lett., 106, 130402 (2011).

11. T.Maudlin, Am.J.Phys, 78, 121 (2010).

12. M.D.Reid, P.D.Drummond, W.P.Bowen et al., Rev. Mod. Phys., 81, 1727 (2009).

13. L.Amico, R.Fazio, A.Osterloh, V.Vedral, Rev. Mod. Phys., 80, 518 (2008).

14. J.M.Raimond, M.Brune, S.Haroche, Rev. Mod. Phys., 73, 565 (2001).

15. W.H.Zurek, Rev. Mod. Phys., 75, 715 (2003).

16. A.Zeilinger, Rev.Mod.Phys., 71, S288 (1999).

17. B.Hensen et al., Nature, 526, 682 (2015).

18. H.Wiseman, Nature, 526, 649 (2015).

19. D.N.Klyshko, Physics-Uspekhi, 158, 327 (1989).

20. D.N.Klyshko, Physics-Uspekhi, 168, 975 (1998).

21. R.Hanbury Brown and R.Q.Twiss, Nature (London) 177, 27 (1956).

22. G.'t Hooft, ArXiv [quant-ph):1308.1007 (2013).

23. G.'t Hooft, ArXiv [quant-ph):1405.1548 (2014). 